Received: 21.03 .2018

Revised: 30.04 .2018

Accepted: 15.06 .2018

DOI: $10.17804 / 2410-9908.2018 .3 .024-032$

\title{
PREDICTION OF RADIATION RESISTANCE OF NANOSIZED COATINGS
}

\author{
O. V. Mkrtychev
}

\begin{abstract}
Branch of Belgorod State Technological University named after V. G. Shukhov in Novorossiysk, Myskhakskoe shosse, 75, Novorossiysk, 353919, Russian federation

(iD https://orcid.org/0000-0002-9619-1029; ه oleg214@ya.ru

\author{
Corresponding author. E-mail: oleg214@ya.ru \\ Address for correspondence: Myskhakskoe shosse, 75, Novorossiysk, 353919, Russian federation
}

In the course of studying laser ablation destruction of various materials, one of the challenges for researchers is the prediction of the radiation strength of such materials. A great interest in laser ablation of thin-film coatings and optical materials (glasses, crystals, optical fiber and other optical elements) is due to their wide application in nanophotonics and optoelectronics for various purposes when they are subjected to high-power laser radiation. The problems of predicting the dynamics of the optical strength of such samples are considered in this paper using Weibull statistical distribution. In this paper, we investigate a method of determining the radiation strength of transparent materials from the results of their laser ablation destruction based on Weibull statistical distribution for predicting the time of their failure-free operation.

Keywords: radiation resistance, radiation strength, laser ablation, Weibull distribution.

\section{References}

1. Wippermann S., He Yu., Vörös M., Galli G. Novel silicon phases and nanostructures for solar energy conversion. Applied Physics Reviews, 2016, no. 3, pp. 040807. DOI: 10.1063/1.4961724.

2. Kotelnikova A.A., Karengin A.G., Mendoza O. Plasmachemical synthesis and evaluation of the thermal conductivity of metal-oxide compounds "Molybdenum-uranium dioxide". AIP Conference Proceedings, 2018, no. 1938, pp. 020015. DOI: 10.1063/1.5027222.

3. Dorosheva I.B., Valeeva A.A., Rempel A.A. Sol-gel synthesis of nanosized titanium dioxide at various $\mathrm{pH}$ of the initial solution. AIP Conference Proceedings, 2017, no. 1886, pp. 020006. DOI: $10.1063 / 1.5002903$.

4. $\quad$ Ling W.Y.L., Schönherr T., Koizumi H. Appl. Phys. Lett., 2017, no. 111, pp. 014101. DOI: $10.1063 / 1.4991713$.

5. Baranov O., Bazaka K., Kersten H., Keidar M., Cvelbar U., Xu S., Levchenko I. Plasma under control: Advanced solutions and perspectives for plasma flux management in material treatment and nanosynthesis. Applied Physics Reviews, 2017, no. 4, pp. 041302. DOI: 10.1063/1.5007869.

6. Smirnov S.V., Smirnova E.O., Veretennikova I.A., Fomin V.M., Filippov A.A., Brusentseva T.A. Studying epoxy resin reinforced with silica dioxide nanoparticles by microindentation. Diagnostics, Resource and Mechanics of materials and structures, 2017, iss. 1, pp. 24-35. DOI: 10.17804/2410-9908.2017.1.024-035. Available at: http://dreamjournal.org/DREAM_Issue_1_2017_Smirnov_S.V._et_al._024_035.pdf

7. Koroleva L.F. Nanoparticulate zirconia-modified solid solutions of aluminum-iron oxides for polishing titanium metal. Diagnostics, Resource and Mechanics of materials and structures, 2015, iss. 1, pp. 90-102. DOI: 10.17804/2410-9908.2015.1.090-102. Available at: http://dreamjournal.org/DREAM_Issue_1_2015_Koroleva_L._F..pdf 
8. Gorkunov E.S. Magnetic evaluation of the structural and phase changes in individual layers of multilayer products. Diagnostics, Resource and Mechanics of materials and structures, 2017, iss. 2, pp. 6-27. DOI: 10.17804/2410-9908.2017.2.006-027. Available at: http://dreamjournal.org/DREAM_Issue_2_2017_Gorkunov_E.S._006_027.pdf

9. Sokolova N.M. The possibility of the energy approach to studying the fracture of metal glass. Deformatsiya i Razrushenie Materialov, 2017, vol. 1, pp. 6-9. (In Russian).

10. Lyubimova O.N., Morkovin A.V., Dryuk S.A. The features of the structure of the glass-steel junction zone in the glass-metal composite production process. Materialovedenie, 2017, vol. 4, pp. 3-7. (In Russian).

11. Kazilin E.E., Konkin S.V., Ivanov L.I., Sinitsyna O.V., Tikhonov A.N., Yaminskii I.V. Creation of composite materials using carbon colloidal solutions obtained by laser technology. Inorganic Materials: Applied Research, 2010, vol. 1, iss. 4, pp. 350-352. DOI: 10.1134/S2075113310040155.

12. Kalin B.A., Yakushin V.L., Dzhumaev P.S., Pol'skii V.I., Golovchanskii I.A., Fedotov V.T., Sevryukov O.N., Suchkov A.N. Development of a method for producing metal materials with a nanostructured surface layer by treatment with high-energy pulsed plasma. Inorganic Materials: Applied Research, 2011, vol. 2, iss. 3, pp. 218-223. DOI: 10.1134/S2075113311030117.

13. Manenkov A.A., Prokhorov A.M. Laser fracture of transparent solids. UFN, 1986, vol. 148, iss. 1, pp. 179-211. (In Russian).

14. Mkrtychev O.V. Analytical study of the energy coefficients of light reflection and refraction. Izvestiya Vysshikh Uchebnykh Zavedeniy. Severo-Kavkazskiy Region. Seriya: Estestvennye Nauki, 2012, vol. 4, pp. 36-38. (In Russian).

15. Anisimov S.I., Lukyanchuk B.S. Selected problems of the laser ablation theory. UFN, 2002, vol. 172, iss. 3, pp. 301-333. (In Russian).

16. Sobol E.N. Phase Transformations and Ablation in Laser-Treated Solids. New York, Wiley, 1995.

17. Lyubov B.Ya., Sobol E.N. In: N.N. Rykalin, ed. Deistvie kontsentrirovanykh potokov energii na materialy [The Effect of Concentrated Energy Flows on Materials]. Moscow, Nauka Publ., 1985, 226 p. (In Russian).

18. Weibull W. Statistical distribution function of wide applicability. Journal of Applied Mechanics, 1951, vol. 18, pp. 293-297.

19. Madjoubi M.A., Bousbaa C., Hamidouche M., Bouaouadja N. Weibull statistical analysis of the mechanical strength of a glass eroded by sand blasting. Journal of the European Ceramic Society, 1999, vol. 19, no. 16, pp. 2957-2962.

20. Ventsel E.S., Ovcharov L.A. Teoriya sluchainykh protsessov i eyo inzhenernye prilozheniya [The Random Process Theory and its Engineering Applications]. Moscow, Vysshaya Shkola Publ., 2000, 383 p. (In Russian).

21. Ivshin V.V. Statistical problems of reliability estimation in the load-strength model for the cases of gamma distribution and Weibull distribution. Vestnik PNIPU. Mekhanika, 1994, vol. 2, iss. 2, pp. 43-49. (In Russian).

22. Tashkinov M.A. A stochastic model of microstructural damage based on the restoration of random field distribution laws in microinhomogeneous media. Vestnik PNIPU. Mekhanika, 2017, vol. 4, pp. 76-91. (In Russian).

23. Kadrik K.A., Mkrtychev O.V. On the dynamics and kinematics of energy interaction with a system of plane-parallel or concentric media. Vestnik Belgorodskogo Gosudarstvennogo Technologicheskogo Universiteta im. V.G. Shukhova, 2014, vol. 1, pp. 238-242. (In Russian).

24. Mkrtychev O.V., Shemanin V.G. Prediction of the optical strength of nanocomposites. $P e$ terburgskiy Zhurnal Elektroniki, 2014, vol. 3, iss. 80, pp. 13-22. (In Russian).

25. Mkrtychev O. V., Shemanin V.G. Laser-ablation destruction of nanofilms on the surface of glass specimens. Izvestiya Ufimskogo Nauchnogo Tsentra RAN, 2015, vol. 2, pp. 5-10. (In Russian). 
26. Mkrtychev O.V., Privalov V.E., Fotiadi A.E., Shemanin V.G. Laser ablation of nanocomposites. Nauchno-Tekhnicheskie Vedomosti Sankt-Peterburgskogo Gosudarstvennogo Politekhnicheskogo Universiteta. Fiziko-Matematicheskie Nauki, 2015, vol. 1, iss. 213, pp. 128-135. (In Russian).

27. Shemanin V.G., Mkrtychev O.V. The optical strength of the glass nanocomposites at laser ablation. Journal of Physics: Conference Series, 2015, vol. 653, iss. 1, pp. 012012.

28. Shemanin V.G., Mkrtychev O.V. Temperature Field under Laser Ablative Destruction of Target at Low Temperatures. Technical Physics, 2018, vol. 63, iss. 5, pp. 623-627. DOI: $10.1134 / \mathrm{S} 106378421805021388$. 
Подана в журнал: 21.03.2018

УДК 543.4

DOI: $10.17804 / 2410-9908.2018 .3 .024-032$

\title{
ПРОГНОЗИРОВАНИЕ ЛУЧЕВОЙ ПРОЧНОСТИ НАНОРАЗМЕРНЫХ ПОКРЫТИЙ
}

\author{
О. В. Мкртычев \\ Филиал ФГБОУ ВО «Белгородский государственный технологический университет им. В.Г. Шухова», \\ Мысхакское шоссе, 75, г. Новороссийск, 353919, Российская Федераџия \\ (iD https://orcid.org/0000-0002-9619-1029; $\otimes$ oleg214@ya.ru \\ Ответственный автор. Электронная почта: oleg214@ya.ru \\ Адрес для переписки: Мысхакское шоссе, 75, г. Новороссийск, 353919, Российская Федерация
}

В ходе исследований лазерного абляционного разрушения различных материалов одной из задач, встающих перед исследователями, является задача прогнозирования лучевой прочности таких материалов. Большой интерес к лазерной абляции нанокомпозитных (тонкопленочные покрытия) и оптических материалов (стекла, кристаллы, оптическое волокно и другие оптические элементы) связан с их широким применением в приборах нанофотоники и оптоэлектроники различного назначения, подвергаясь при этом мощному лазерному излучению. Вопросы прогнозирования динамики оптической прочности таких образцов рассматриваются в этой статье с применением статистического распределения Вейбулла-Гнеденко. $\mathrm{B}$ настоящей статье исследуется методика определения лучевой прочности прозрачных материалов по результатам их лазерного абляционного разрушения на основе статистического распределения Вейбулла-Гнеденко для прогнозирования времени их безотказной работы.

Ключевые слова: лучевая прочность, лазерная абляция, распределение Вейбулла-Гнеденко.

\section{1. Введение}

Прочность материалов под действием мощного излучения является важным вопросом, стоящим перед развитием многих областей современных технологий, включая нанофотонику, оптическое материаловедение [1-5]. К таким материалам относятся керамические материалы и стекло или соединения на их основе, например, в стеклометаллокомпозитных материалах, которые часто употребляются в различных отраслях [6-9]. Такие материалы часто упрочняют путем введения специальных добавок или путем нанесения на его поверхность тонкопленочных покрытий [10-12]. Среди различных видов взаимодействия в последнее время все больший практический и теоретический интерес вызывает разрушение поверхности материалов, подвергающихся воздействию мощного лазерного импульса и (или) плазмы, образующейся при этом $[13,14]$. Согласно сложившимся представлениям тепловой модели лазерного абляционного разрушения [13, 15-17], разрушение происходит в результате разогрева случайно распределенных дефектов материала. Так как материалы не могут представлять идеально однородную среду, то в них на поверхности и в объеме располагаются дефекты различной природы и различных размеров. Теория прочности материалов с таким случайно распределенным в материале набором дефектов допускает, что прочность слоев материала на поверхности значительно меньше прочности материала в объеме [18-22]. Ввиду случайности распределения дефектов прочность материала является статистической физической величиной. При рассмотрении лазерного абляционного разрушения авторы применяли статистическое распределение Вейбулла-Гнеденко (в англоязычной литературе «распределение Вейбулла»), которое часто применяется для определения прочности хрупких 
материалов [например 20]. Авторы статей [23-28] провели ряд исследований стеклянных материалов, покрытых наноразмерными покрытиями из разного количества пленок, полученных из диоксидов кремния и титана методом золь-гель-технологий. В результате этих исследований разработана методика прогнозирования динамики лучевой прочности наноразмерных покрытий под действием мощного импульсного излучения.

\section{2. Методы исследования}

Рассмотрим один из видов лучевой прочности, а именно, прочности материала при разрушении вследствие разогрева микродефектов. Ввиду случайного распределения дефектов по облучаемой поверхности этот вид разрушения исследуют с применением статистических методов. Для количественного анализа лучевой прочности хрупких материалов используем модель Вейбулла-Гнеденко, основанную на принципе «наиболее слабого звена». Если использовать только интегральную характеристику облучающего импульса - плотность

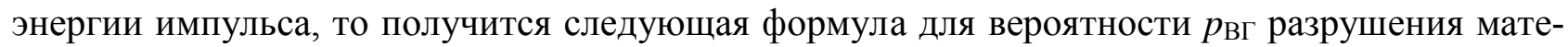
риала в модели Вейбулла-Гнеденко:

$$
p_{\mathrm{B} \Gamma}(F)= \begin{cases}1-\exp \left\{-\ln 2\left[\sum_{j=1}^{k}\left(\frac{F}{F_{0,5}}\right)^{m_{j}}\right]\right\}, & F>0, \\ 0, & F \leq 0,\end{cases}
$$

где $F$ - плотность энергии падающего излучения; $k$ - общее количество разных видов дефекта; $F_{0,5}$ - пробойная плотность энергии, для которой экспериментальная вероятность пробоя равна $p_{\text {эксп }}=0,5 ; m_{j}$ - модуль Вейбулла для данного $j$-го вида дефекта. Предполагается, что в гомогенном материале случайно расположены $k$ типов различных дефектов, которые действуют независимо друг от друга.

В случае однократно облученной мишени, при условии, что вероятность лазерной абляции определяется дефектами одного рода на поверхности образца, вероятность разрушения описывается функцией

$$
p(F)= \begin{cases}1-\exp \left\{-\ln 2\left[\left(\frac{F}{F_{0,5}}\right)^{m_{j}}\right]\right\}=1-e^{-\rho(F) A}, & F>0 \\ 0, & F \leq 0\end{cases}
$$

где $\rho(F)=k F^{m}$ - средняя поверхностная концентрация дефектов; $A$ - объем области, подвергнутой воздействию лазерного излучения с плотностью энергии $F$. Лучевая прочность покрытия образца будет в этом случае иметь вид:

$$
q(F)=1-p(F)=\exp \left[-\ln 2\left(\frac{F}{F_{0,5}}\right)^{m}\right]
$$

Например, для одного из исследованных образцов лучевая прочность в микросекундном диапазоне (рис. 1) равна

$$
q(F)=\exp \left\{-\ln 2\left(\frac{F}{100,17}\right)^{7,74}\right\},
$$

а в наносекундном диапазоне: 


$$
q(F)=\exp \left\{-\ln 2\left(\frac{F}{30,21}\right)^{15,65}\right\}
$$

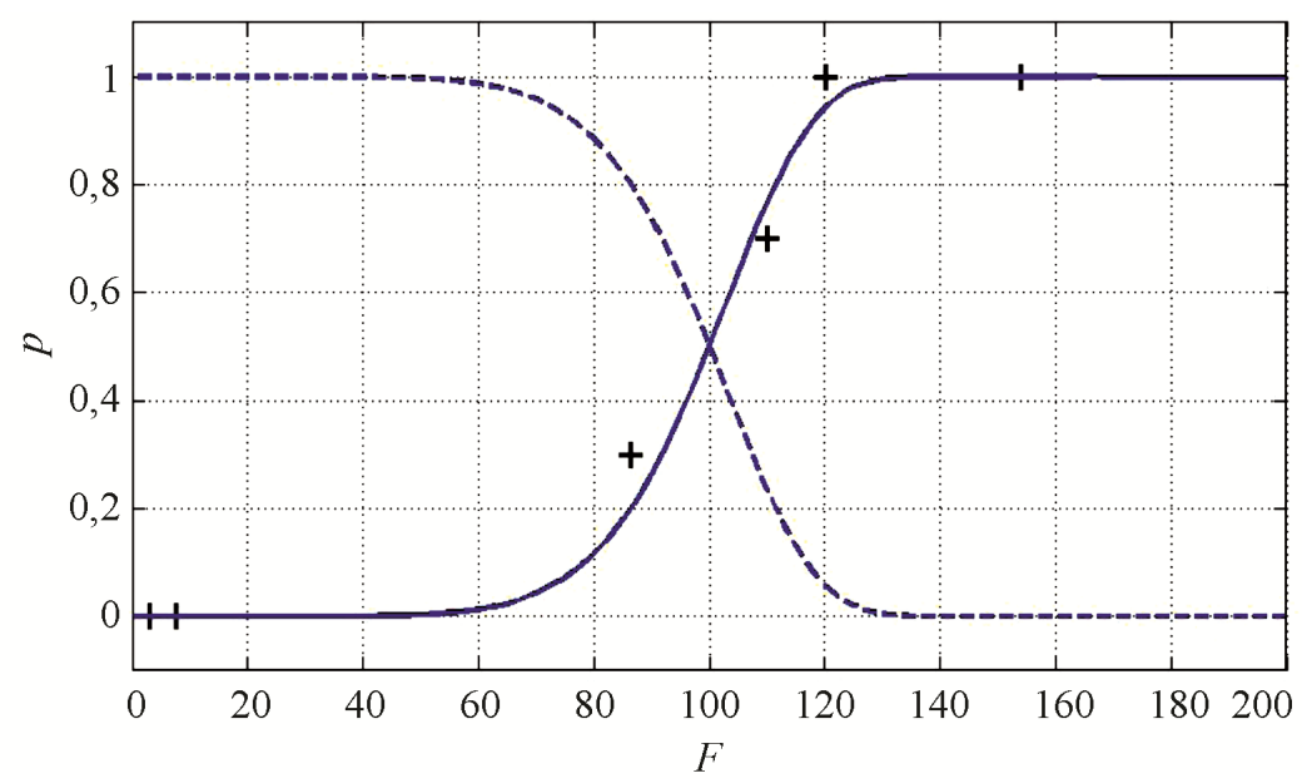

Рис. 1. График интегральной функции распределения $p(F)$ в микросекундном диапазоне: точки - экспериментальные данные; сплошная кривая - аппроксимация функцией распределения Вейбулла-Гнеденко; штриховая линия - оптическая прочность.

\section{3. Результаты и их обсуждение}

Эксперименты проводились на установке, схема которой представлена на рис. 2. Сначала каждую точку поверхности исследуемого образца подвергают однократному облучению импульсом излучения с заданной плотностью энергии $F_{i}(i=1,2, \ldots n$, где $n$ - количество значений плотности энергии пучка) и регистрируют возникновение оптического пробоя, индуцированного лазерным излучением. После этого процесс повторяют в другой точке и после проведения цикла измерения вычисляют экспериментальное значение вероятности $p_{\text {эксп }}\left(F_{i}\right)$. Далее по этим экспериментальным данным строят кривую вероятности разрушения поверхности образца, (рис. 1). По полученной кривой вероятности определяют две величины, описывающие разрушение поверхности материала. Первая величина - это пороговое значение плотности энергии разрушения, которое принимаем равным значению $F_{0,5}$ плотности энергии при вероятности $p=0,5[24,25]$. Вторая величина - это модуль Вейбулла $m$, который вычисляется аналитически. Для этого создается сетка шаблонных функций $p_{\text {шабл }}$, минимизируя величину

$$
\sigma=\sqrt{\frac{\sum_{i=1}^{N_{F}}\left\{p_{\text {шабл }}-p_{\text {эксп }}\right\}^{2}}{N_{F}-1}},
$$

где $\sigma$ - среднеквадратичное отклонение вероятности $p_{\text {ВГ }}$, вычисленной по (5), от экспериментальной вероятности $p_{\text {эксп; }} N_{F}$ - общее количество различных плотностей энергии, при которых проводилось облучение материала в эксперименте, получаем искомую функциональную зависимость вероятности разрушения $p^{*}$ образца от плотности энергии $F$ падающего излучения 


$$
p^{*}= \begin{cases}1-\exp \left\{-\ln 2\left[\sum_{j=1}^{K^{*}}\left(\frac{F}{F_{0,5}}\right)^{M_{j}^{*}}\right]\right\}, & F>0 \\ 0, & F \leq 0\end{cases}
$$

со значениями $K^{*}$ - число различных видов дефектов; $M^{*}$ - модуль Вейбулла соответственного вида дефекта; $F_{0,5}$ определен выше в тексте.

После этого остается выражение для зависимости лучевой прочности $Q$ поверхности материала от плотности энергии $F$ падающего излучения получает вид:

$$
Q(F)= \begin{cases}\exp \left\{-\ln 2\left[\sum_{j=1}^{K^{*}}\left(\frac{F}{F_{0,5}}\right)^{M_{j}^{*}}\right]\right\}, & F>0 \\ 0, & F \leq 0\end{cases}
$$

где $Q$ - лучевая прочность облучаемого объема материала.

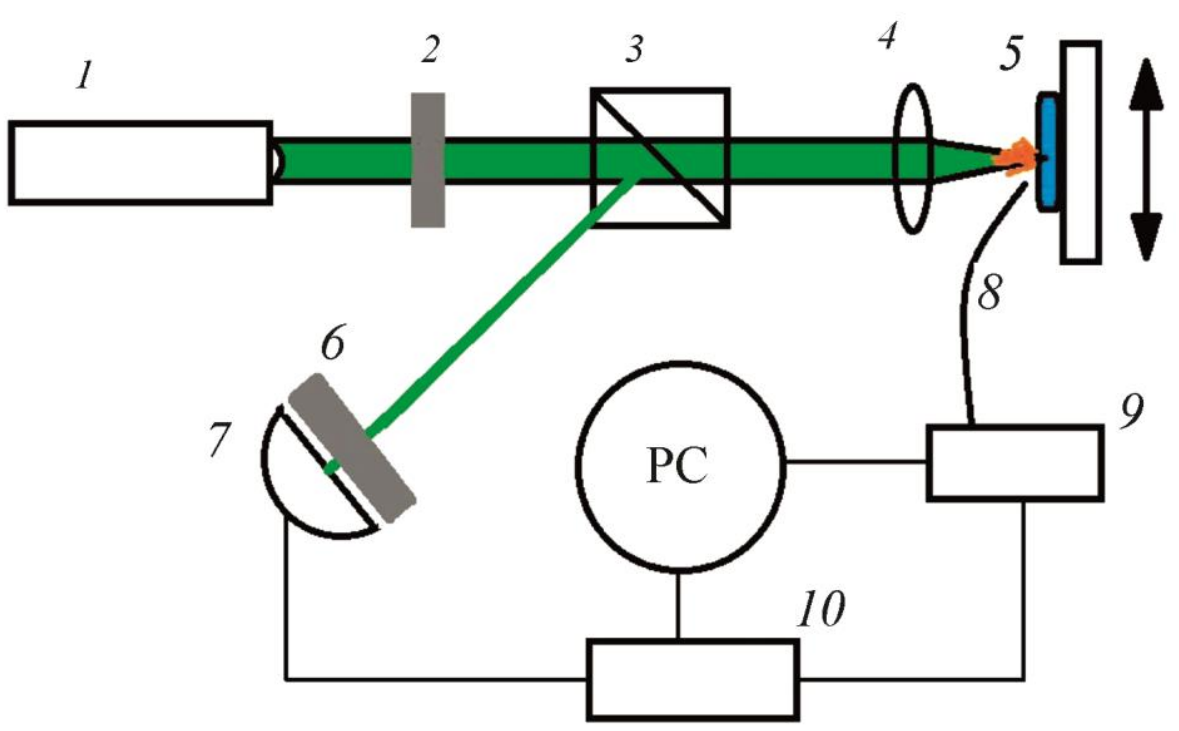

Рис. 2. Схема экспериментальной установки: 1 - источник излучения; 2 - нейтральный светофильтр для изменения энергии излучения; 3 - стеклянная пластина; 4 - фокусирующая линза; 5 - образец; 6 - светофильтр ИКС-1; 7 - фотодиод; 8 - световод;

9 - микро-спектрометр FSD-8; 10 - управляемая ПК линия задержки

\section{4. Выводы}

Ряд исследований и экспериментов свидетельствуют, что погрешность вычислений, установленная на основании сравнения экспериментальных данных и измерений для значений параметров с $j=1,2,3$ по (4) с доверительной вероятностью 0,9, не превышает $18 \%$ $[14,23-28]$. Таким образом, в результате исследований прочности материалов силовой оптики, проведенных авторами для учета влияния различных физических факторов на лучевую прочность, разработана методика прогнозирования прочностных свойств поверхности оптических материалов, с использованием статистического распределения Вейбулла-Гнеденко.

\section{Список литературы}

1. Novel silicon phases and nanostructures for solar energy conversion / S. Wippermann, Yu. He, M. Vörös, G. Galli // Applied Physics Reviews. - 2016. - No. 3. - P. 040807. DOI: $10.1063 / 1.4961724$. 
2. Kotelnikova A. A., Karengin A. G., Mendoza O. Plasmachemical synthesis and evaluation of the thermal conductivity of metal-oxide compounds "Molybdenum-uranium dioxide" // AIP Conference Proceedings. - 2018. - No. 1938. - P. 020015. - DOI: 10.1063/1.5027222.

3. Dorosheva I. B., Valeeva A. A., Rempel A. A. Sol-gel synthesis of nanosized titanium dioxide at various $\mathrm{pH}$ of the initial solution // AIP Conference Proceedings. - 2017. - No. 1886. P. 020006. - DOI: 10.1063/1.5002903.

4. $\quad$ Ling W. Y. L., Schönherr T., Koizumi H. Discharge characteristics of an ablative pulsed plasma thruster with non-volatile liquid propellant // Appl. Phys. Lett. - 2017. - No. 111. P. 014101. - DOI: 10.1063/1.4991713.

5. Plasma under control: Advanced solutions and perspectives for plasma flux management in material treatment and nanosynthesis / O. Baranov, K. Bazaka, H. Kersten, M. Keidar, U. Cvelbar, S. Xu, I. Levchenko // Applied Physics Reviews. - 2017. - No. 4. - P. 041302. DOI: $10.1063 / 1.5007869$.

6. Studying epoxy resin reinforced with silica dioxide nanoparticles by microindentation / S. V. Smirnov, E. O. Smirnova, I. A. Veretennikova, V. M. Fomin, A. A. Filippov, T. A. Brusentseva // Diagnostics, Resource and Mechanics of materials and structures. - 2017. Iss. 1. - P. 24-35. - DOI: 10.17804/2410-9908.2017.1.024-035. - URL: http://dreamjournal.org/DREAM_Issue_1_2017_Smirnov_S.V._et_al._024_035.pdf

7. Koroleva L. F. Nanoparticulate zirconia-modified solid solutions of aluminum-iron oxides for polishing titanium metal // Diagnostics, Resource and Mechanics of materials and structures. 2015. - Iss. 1. - P. 90-102. - DOI: 10.17804/2410-9908.2015.1.090-102. - URL: http://dreamjournal.org/DREAM_Issue_1_2015_Koroleva_L._F..pdf

8. Gorkunov E. S. Magnetic evaluation of the structural and phase changes in individual layers of multilayer products // Diagnostics, Resource and Mechanics of materials and structures. - 2017. Iss. 2. - P. 6-27. - DOI: 10.17804/2410-9908.2017.2.006-027. - URL: http://dreamjournal.org/DREAM_Issue_2_2017_Gorkunov_E.S._006_027.pdf

9. Соколова Н. М. Возможность энергетического подхода к изучению процессов разрушения металлических стекол // Деформация и разрушение материалов. - 2017. - Т. 1. - С. 6-9.

10. Любимова О. Н., Морковин А. В., Дрюк С. А. Особенности структуры зоны соединения стекла и стали в технологии получения стеклометаллокомпозита // Материаловедение. 2017. - T. 4. - C. 3-7.

11. Creation of composite materials using carbon colloidal solutions obtained by laser technology / E. E. Kazilin, S. V. Konkin, L. I. Ivanov, O. V. Sinitsyna, A. N. Tikhonov, I. V. Yaminskii // Inorganic Materials: Applied Research. - 2010. - Vol. 1, iss. 4. - P. 350-352. DOI: $10.1134 / \mathrm{S} 2075113310040155$.

12. Development of a method for producing metal materials with a nanostructured surface layer by treatment with high-energy pulsed plasma / B. A. Kalin, V. L Yakushin., P. S. Dzhumaev, V. I. Pol'skii, I. A. Golovchanskii, V. T. Fedotov, O. N. Sevryukov, A. N. Suchkov // Inorganic Materials: Applied Research. - 2011. - Vol. 2, iss. 3. - P. 218223. - DOI: 10.1134/S2075113311030117.

13. Маненков А. А., Прохоров А. М. Лазерное разрушение прозрачных твердых тел // УФН. - 1986. - Т. 148, вып. 1. - С. 179-211.

14. Мкртычев О. В. Аналитическое исследование энергетических коэффициентов отражения и преломления света // Известия высших учебных заведений. Северо-Кавказский регион. Серия: Естественные науки. - 2012. - Т. 4. - С. 36-38.

15. Анисимов С. И., Лукьянчук Б. С. Избранные задачи теории лазерной абляции // УФН. - 2002. - Т. 172, вып. 3. - С. 301-333.

16. Sobol E. N. Phase Transformations and Ablation in Laser-Treated Solids. - New York, Wiley, 1995. 
17. Любов Б. Я., Соболь Э. Н. // Воздействие концентрированных потоков энергии на материалы: сборник статей / АН СССР, ин-т металлургии им. А.А. Байкова; отв. ред. Н. Н. Рыкалин - М. : Наука, 1985. - С. 226.

18. Weibull W. Statistical distribution function of wide applicability // Journal of Applied Mechanics. - 1951. - Vol. 18. - P. 293-297.

19. Weibull statistical analysis of the mechanical strength of a glass eroded by sand blasting / M. A. Madjoubi, C. Bousbaa, M. Hamidouche, N. Bouaouadja // Journal of the European Ceramic Society. - 1999. - Vol 19, no. 16. - P. 2957-2962.

20. Вентцель Е. С., Овчаров Л. А. Теория случайных процессов и ее инженерные приложения. - М. : Высшая школа, 2000. - 383 с.

21. Ившин В. В. Статистические задачи оценивания надежности в модели «нагрузкапрочность» в случаях гамма-распределения и распределения Вейбулла // Вестник Пермского национального исследовательского политехнического университета. Механика. - 1994. № 2 (2). - С. 43-49.

22. Ташкинов М. А. Стохастическая модель микроструктурного разрушения на основе восстановления законов распределений случайных полей в микронеоднородных средах // Вестник Пермского национального исследовательского политехнического университета. Механика. - 2017. - № 4. - С. 76-91.

23. Кадрик К. А., Мкртычев О. В. К динамике и кинематике энергетического взаимодействия с системой плоскопараллельных или концентрических сред // Вестник Белгородского государственного технологического университета им. В. Г. Шухова. - 2014. - Т. 1. - С. 238-242.

24. Мкртычев О. В., Шеманин В. Г. Прогнозирование оптической прочности нанокомпозитов // Петербургский журнал электроники. - 2014. - Т. 3, вып. 80. - С. 13-22.

25. Мкртычев О. В., Шеманин В. Г. Лазерное абляционное разрушение нанопленок на поверхности стеклянных образцов // Известия Уфимского научного центра РАН. - 2015. - Т. 2. C. $5-10$.

26. Мкртычев О. В., Привалов В. Е., Фотиади А. Э., Шеманин В. Г. Лазерная абляция нанокомпозитов // Научно-технические ведомости Санкт-Петербургского государственного политехнического университета. Физико-математические науки. - 2015. - Т. 1, вып. 213. C. $128-135$.

27. Shemanin V. G., Mkrtychev O. V. The optical strength of the glass nanocomposites at laser ablation // Journal of Physics: Conference Series. - 2015. - Vol. 653, iss. 1. - P. 012012.

28. Shemanin V. G., Mkrtychev O. V. Temperature Field under Laser Ablative Destruction of Target at Low Temperatures // Technical Physics. - 2018. - Vol. 63, iss. 5. - P. 623-627. DOI: $10.1134 / \mathrm{S} 106378421805021388$. 